\title{
Light yield of an undoped Csl crystal coupled directly to a photomultiplier tube at 77 Kelvin
}

\author{
J. Liu ${ }^{a, 1}$ M. Yamashita ${ }^{b, c}$ A. K. Soma ${ }^{a}$ \\ ${ }^{a}$ Department of Physics, University of South Dakota, \\ 414 East Clark Street, Vermillion, South Dakota 57069, USA \\ ${ }^{b}$ Kamioka Observatory, Institute for Cosmic Ray Research, University of Tokyo, \\ Higashi-Mozumi, Kamioka, Hida, Gifu, 506-1205, Japan \\ ${ }^{c}$ Kavli Institute for the Physics and Mathematics of the Universe (WPI), University of Tokyo, \\ Kashiwa, Chiba, 277-8582, Japan \\ E-mail: jing.liu@usd.edu
}

ABSTRACT: A light yield of $20.4 \pm 0.8$ photoelectrons $/ \mathrm{keV}$ was achieved with an undoped CsI crystal coupled directly to a photomultiplier tube at 77 Kelvin. This is by far the largest yield in the world achieved with CsI crystals. An energy threshold that is several times lower than the current dark matter experiments utilizing $\mathrm{CsI}(\mathrm{Tl})$ crystals may be achievable using this technique. Together with novel CsI crystal purification methods, the technique may be used to improve the sensitivities of dark matter and coherent elastic neutrino-nucleus scattering experiments. Also measured were the scintillation light decay constants of the undoped CsI crystal at both room temperature and 77 Kelvin. The results are consistent with those in the literature.

KeYwords: Dark Matter detectors, Cryogenic detectors, Scintillators, scintillation and light emission processes (solid, gas and liquid scintillators), Photon detectors for UV, visible and IR photons (vacuum) (photomultipliers, HPDs, others)

ARXIV EPRINT: 1608.06278

\footnotetext{
${ }^{1}$ Corresponding author.
} 


\section{Contents}

1 Introduction 1

2 Experimental setup 3

3 Single-photoelectron response of the PMT 4

$\begin{array}{lll}4 & \text { Energy calibration of the system } & 6\end{array}$

$\begin{array}{llr}5 & \text { Light yield of the system } & 8\end{array}$

6 Quantum efficiency of the PMT $\quad 8$

$\begin{array}{lll}7 & \text { Scintillation decay constants of the crystal } & 10\end{array}$

8 Impacts on rare-event search experiments 13

9 Conclusion 14

\section{Introduction}

The thallium doped sodium iodide and cesium iodide at room temperature, $\mathrm{NaI} / \mathrm{CsI}(\mathrm{Tl})$ in short hereafter, are widely used in direct dark matter search experiments [1-3], due to their high scintillation light yields $(\sim 50$ photons $/ \mathrm{keV})$ and relatively low costs. The DAMA experiment observed an annual modulation signal in the 2-6 keV region in their $\mathrm{NaI}(\mathrm{Tl})$ scintillators [2]. If it is interpreted with the standard dark matter theory, the observation conflicts with results from experiments using different target materials [3-6]. Many experiments have been proposed to verify the DAMA result using the same material in different environments, including ANAIS [7], DM-Ice [1], KIMS-NaI [8], COSINE [9] and SABRE [10] etc. One of the main difficulties is to reduce the radioactive contamination, ${ }^{40} \mathrm{~K}$ for instance, in NaI powder. The $\sim 3 \mathrm{keV}$ X-rays/Auger electrons from ${ }^{40} \mathrm{Ar}$, the daughter of ${ }^{40} \mathrm{~K}$ electron-capture decay, are potential background for dark matter search. A higher light yield of $\mathrm{NaI} / \mathrm{CsI}$ crystals will lead to a lower energy threshold and better energy resolution. The analysis window can be moved from $2-6 \mathrm{keV}$ to a lower energy region, 1-2 keV for example, where more dark matter signal is expected and the contamination from the $\sim 3 \mathrm{keV}$ peak is negligible.

Sodium doped CsI crystals are used in the CosI experiment [11] to detect coherent elastic neutrino-nucleus scattering (CEvNS) events. The CEvNS process is important in the evolution of astronomical objects [12]. It can also be used to probe non-standard neutrino interactions [13], sterile neutrinos [14] and nuclear structures [15, 16], as well as to monitor the activity of a reactor [17]. Many experiments along with CosI have been performed or proposed to detect it, for example, TEXONO [18], CoGeNT using Ge detectors, RED [19] using liquid xenon, and COHERENT [11, 20] using all of them, etc. The major difficulty 
is that the energies of recoiled nuclei are so small that detectors with extremely low energy thresholds are needed.

A straight forward way to lower the energy threshold of a scintillator is to increase its light yield. It was observed that the light yields of undoped NaI/CsI increase rapidly when temperature goes down, and reach the highest point around liquid nitrogen temperature (77 Kelvin at one atmospheric pressure) [21-45]. A theoretical explanation based on quantum statistics was given by Hsu et al. in 1966 [28]. The observed photons varied with the purity of crystals and light readout methods. Nevertheless, all measurements gave similar or higher yields than those of $\mathrm{NaI} / \mathrm{CsI}(\mathrm{Tl})$ at room temperature. The highest ones $[21,24,39,41]$ almost reach the theoretical limit deduced from the band gap energy.

Given such high yields, however, the application of undoped NaI/CsI at 77 Kelvin is rare, because the resistivity of the photo-cathode of a photomultiplier tube (PMT), becomes too high at 77 Kelvin. Light guides coupled to PMTs at room temperature were used to circumvent this problem. But light losses in guides and between multiple optical interfaces cancel out the high yield.

The avalanche photodiode (APD) has been used to read out light from undoped $\mathrm{NaI} / \mathrm{CsI}$ at low temperatures [41, 44]. Since it is operated at linear amplification mode, the amplification factor is only at the order of 100 to 1000, much smaller than that of a PMT. This limitation makes it impossible to be used to lower the energy threshold for dark matter or CEvNS detection.

Silicon photomultiplier (SiPM) is basically an array of APDs working in Geiger mode. It can be used to detect individual photoelectrons. It is very noisy at room temperature, but quiet at 77 Kelvin. All make it very suitable as a readout sensor for undoped NaI/CsI at 77 Kelvin. However, there are some issues [46] limiting its usage in dark matter or CEvNS detection. First of all, the after pulse rate rises very fast below 120 Kelvin. Secondly, the quantum efficiency (QE) reaches the highest point around $500 \mathrm{~nm}$ to $600 \mathrm{~nm}$, while the wavelengths of undoped NaI/CsI at 77 Kelvin peak at around $313 \mathrm{~nm}$ [44] and $340 \mathrm{~nm}$ [34, 36, 38], respectively. Third, there are optical cross talks between individual APDs in the array. Last, the linear energy response is only available if the number of hit APDs is below a certain percentage of the total number of APDs. Nevertheless, it is an attractive option. The issues may be addressed in a separate paper.

It was tried by Persyk et al. in 1980 to couple an undoped NaI directly to a PMT with metal coated beneath its photo-cathode to keep its resistivity low at 77 Kelvin [33]. However the PMT (Hamamatsu R878) had very low QE for the $300 \mathrm{~nm}$ scintillation light emitted from an undoped $\mathrm{NaI}$ at 77 Kelvin.

The performance of PMTs at cryogenic temperatures has been improved gradually over the years. Recently, Hamamatsu Photonics K.K. developed PMT R8778MODAY(AR) [47] based on PMT R8778 for the XMASS experiment [48]. Its QE is approaching 30\% even at 77 Kelvin. Several other PMTs are also available from Hamamatsu to operate at 77 Kelvin, for example, R6041-506 and R11065, etc [49]. By coupling undoped NaI/CsI crystals directly to those PMTs at 77 Kelvin, a light collecting efficiency higher than previous measurements is expected. An energy threshold lower than those of DAMA and KIMS can be achieved.

An experiment was performed to verify this idea. The light yield of the whole system, including an undoped CsI crystal and a PMT R8778MODAY(AR), was measured at both room temperature and 77 Kelvin and reported here. The data from an undoped NaI crystal is under processing and will be published separately. 


\section{Experimental setup}

Figure 1 shows the schematic of the experimental setup (not to scale). Both the PMT and the crystal was housed in a $50 \mathrm{~cm}$ long stainless steel chamber, which was vacuumed down to about $10^{-6}$ mbar and then filled with nitrogen gas up to $0.17 \mathrm{MPa}$ (absolute). The chamber was placed inside an open dewar so that it could be cooled down by pouring liquid nitrogen into the dewar. The temperature profile inside the chamber was controlled by varying the liquid nitrogen level and monitored by three PT100 temperature sensors. One sensor was pressed against the bottom Con-

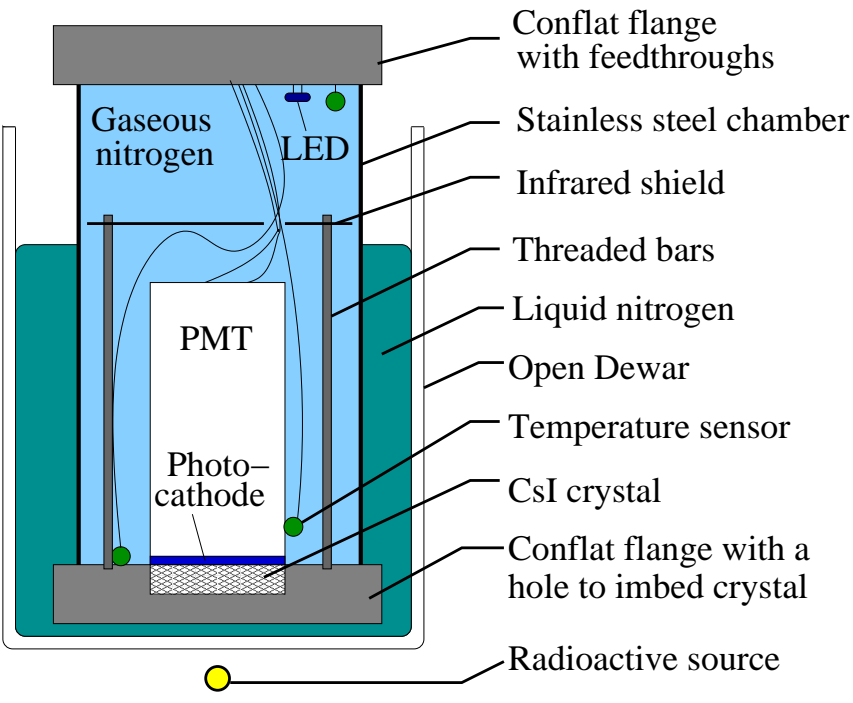

Figure 1. Schematic experimental setup (not to scale). flat flange of the chamber, another was mounted to the side surface of the PMT close to the photo-cathode, the last one was hanging right below the top Conflat flange, close to two LEDs used to calibrate the PMT light response. The light emission spectra of the LEDs were almost Gaussian and peaked at $420 \mathrm{~nm}$ and $300 \mathrm{~nm}$, respectively. All electronic and gas feedthroughs were welded into a 6-inch Conflat flange on the top of the chamber.

The undoped cylindrical CsI crystal has a height of $1 \mathrm{~cm}$ and a radius of $1 \mathrm{inch}$. Its top and bottom surface were nicely polished. The side surface was roughly polished as shown in the left most picture in figure 2. Its side and bottom surfaces were wrapped with Teflon tapes to reflect scintillation light. The crystal was embedded into a hole at the center of a customized 6-inch Conflat flange at the bottom of the chamber. The dimensions of the hole were slightly larger than those of the crystal so that the Teflon tape wrapped crystal could be easily placed in as shown in the middle picture of figure 2. The PMT was mounted upside down with its $\sim 2 \mathrm{~mm}$ thick quartz window tightly pushed against the top surface of the crystal to ensure good optical contact without glue, as shown in the right most picture in figure 2. The photo-cathode was on the inner surface of the window. Optical photons from the LEDs could reach the photo-cathode through the side of the quartz window.

Most of the assembly process of the system was done in a glove box flushed with dry nitrogen gas. The relative humidity was below $3 \%$ at $18^{\circ} \mathrm{C}$ during the process. Limited by the size of the glove box, the connection of cables to the top flange was done outside the glove box with a relative humidity around $45 \%$.

The PMT photo-cathode was grounded, the anode was biased to 1,200 V (recommended by Hamamatsu Photonics K.K.) for the measurements at room temperature. The standard cathode-grounding high-voltage dividing circuit was used. The signal coming out of the circuit was fed to a fan-in/fan-out NIM module. One copy was sent to a standard NIM discriminator to generate the trigger signal, the other was sent to a CAEN V1751 waveform digitizer with a sampling rate of $1 \mathrm{GHz}$ and a bandwidth of $500 \mathrm{MHz}$. The dynamic range of the digitizer is $1 \mathrm{~V}$, the resolution is 10 bits. One digital count given by the Analog- 

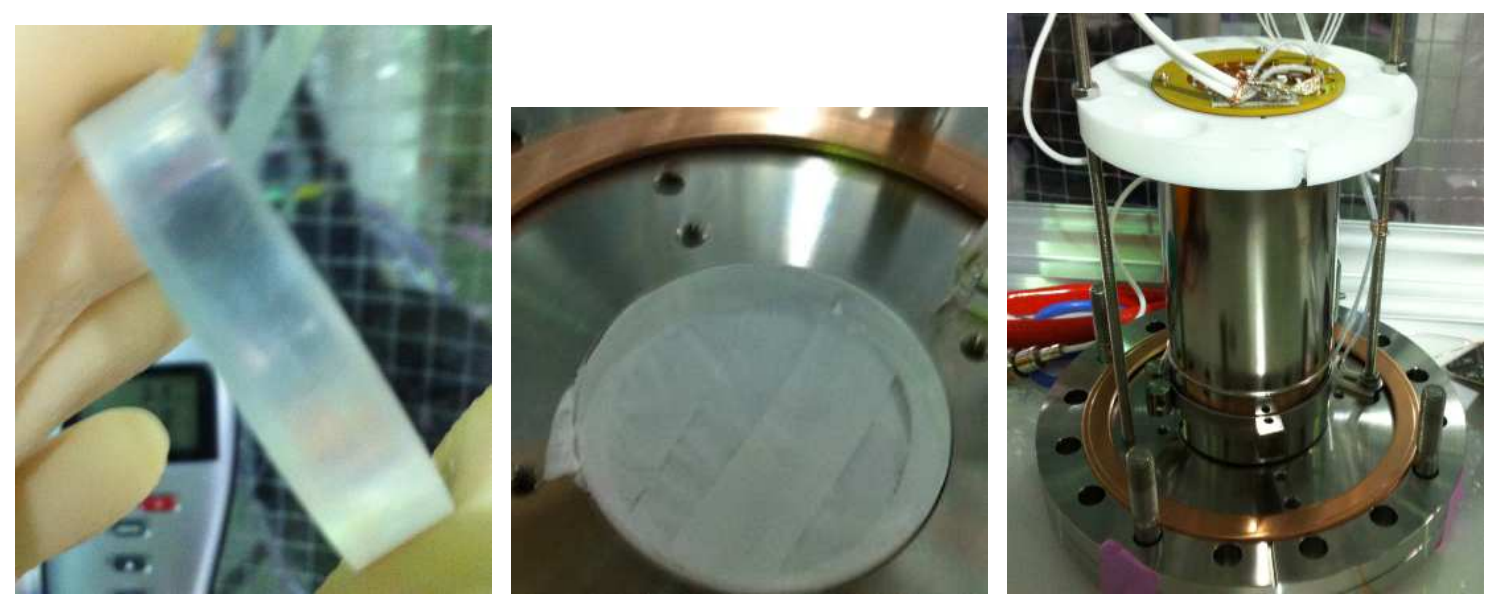

Figure 2. Left: undoped cylindrical CsI crystal with its side surfaces roughly polished. Middle: the crystal embedded into the customized Conflat flange with its bottom and side surfaces wrapped with Teflon tape. Right: PMT mounted on top of the crystal with its photo-cathode facing downward.

to-Digital Converter (ADC count) hence represents $1 \mathrm{~V} / 2^{10}=0.98 \mathrm{mV}$. The digitizer was triggered externally by either the discriminator or a TTL clock signal, which was also used to trigger the LED light emission.

A slightly lower voltage, 1,020 V, was used for the measurements at 77 Kelvin, since the scintillation signal became so strong that the PMT gain had to be lowered to avoid saturation. The electron collection efficiency was measured to be the same as that at 1,200 V. A Phillips Scientific NIM Model 776 had to be used to amplify the PMT signal by a factor of 10 before it was sent to the digitizer in the measurement of the PMT's single photoelectron $(\mathrm{PE})$ response at 77 Kelvin, otherwise the single PE distribution could not be clearly separated from the pedestal noise distribution.

\section{Single-photoelectron response of the PMT}

A $500 \mathrm{~Hz}$ TTL clock signal was used to trigger both the waveform digitizer and the LED power supply. An $800 \mathrm{~ns}$ long waveform was recorded after each trigger. The intensities of the LEDs were tuned such that only one or zero photon struck the PMT photo-cathode during a data acquisition window most of the time.

The left plot of figure 3 shows 50 consecutive PMT waveforms acquired at 77 Kelvin, one is colored in red for a better display of a single PE pulse. The pulse in a waveform, if there are any, is distributed within $(125,165)$ ns. The integration of each waveform was performed from $125 \mathrm{~ns}$ to $165 \mathrm{~ns}$. Its distribution is shown in the right plot of figure 3 . The blue Gaussian function was used to fit the pedestal distribution around zero. The red Gaussian function was used to fit the single PE peak. The green and magenta Gaussian functions were used to fit the second and the third PE distributions, respectively. The widths of the second and the third PE peaks were fixed to that of the single PE peak, and their respective means were fixed to 2 and 3 times of that of the single PE peak.

The measurement was repeated several times. The results are summarized in Table 1. The means of the single PE distributions, $M_{1 \mathrm{PE}}$ (divided by 10 if the amplifier was used), are listed in the last column of the table.

Several things in table 1 are worthy of notice. First, $M_{1 \mathrm{PE}}$ decreases as the applied high voltage decreases. This is expected from the working principle of a PMT. Second, given the 

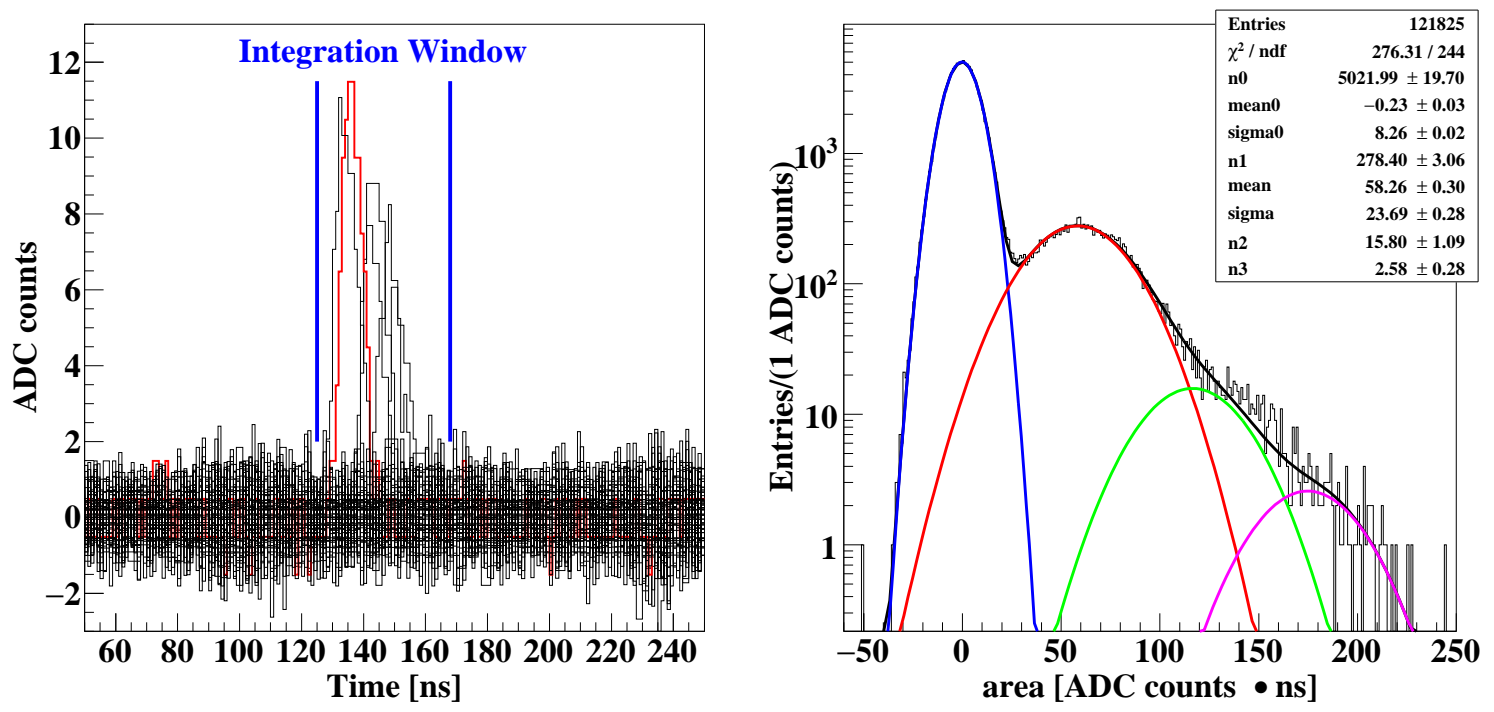

Figure 3. Left: 50 consecutive waveforms overlapped with each other. Right: single PE response of the PMT at a bias voltage of $1,020 \mathrm{~V}$ with an external amplifier (amplification factor: 10).

Table 1. Summary of PMT single PE response measurements.

\begin{tabular}{c|c|c|c|c}
\hline $\begin{array}{c}\text { Temperature } \\
\left.\text { of PMT [ }{ }^{\circ} \mathrm{C}\right]\end{array}$ & $\begin{array}{c}\text { Temperature } \\
\left.\text { of LED [ }{ }^{\circ} \mathrm{C}\right]\end{array}$ & $\begin{array}{c}\text { PMT high } \\
\text { voltage [V] }\end{array}$ & $\begin{array}{c}\text { Wavelength } \\
\text { of LED [nm] }\end{array}$ & $\begin{array}{c}\text { Mean of single PE distri- } \\
\text { bution [ADC counts·ns] }\end{array}$ \\
\hline-191.4 & 8.6 & 1,020 & 470 & $5.849 \pm 0.030$ \\
-191.4 & 8.8 & 1,020 & 300 & $5.489 \pm 0.077$ \\
-192.7 & 17.5 & 1,020 & 300 & $5.068 \pm 0.083$ \\
18.3 & 17.9 & 1,020 & 300 & $3.835 \pm 0.041$ \\
18.1 & 17.8 & 1,200 & 300 & $16.637 \pm 0.294$ \\
-192.7 & 17.5 & 1,200 & 300 & $23.700 \pm 0.471$ \\
\hline
\end{tabular}

same high voltage, $M_{1 \mathrm{PE}}$ increases as the temperature decreases. This may have several causes, including changes of the size, location and properties of PMT dinodes, as well as the resistances of the resistors in the PMT high voltage distributing circuit over temperature. Third, three measurements at $1,020 \mathrm{~V}$ in liquid nitrogen give different $M_{1 \mathrm{PE}}$. The standard deviation is $5.9 \%$. This is too large to be explained by the statistical uncertainties listed in the table. It can neither be explained by the difference of the LED wavelength, which can only affect the QE instead of the PMT gain. The reason is still under investigation. The mean of the first three measurements with $5.9 \%$ uncertainty, $\bar{M}_{1 \mathrm{PE}}=5.468 \pm 0.32$, was used to convert (ADC counts - ns) to the average number of PE in the energy calibration measurements at 77 Kelvin using the following equation,

$$
(\text { number of PE })=(\mathrm{ADC} \text { counts } \cdot \mathrm{ns}) / \bar{M}_{1 \mathrm{PE}} \text {. }
$$

$M_{1 \mathrm{PE}}$ shown in the second last line in table 1 was used as the conversion factor for the energy calibration measurements at room temperature.

$M_{1 \mathrm{PE}}$ can be measured with another method. The number of PE collected from a photocathode obeys the Poisson distribution given a fixed intensity of a light source. According to the central limit theorem, when the expected value $\lambda_{\mathrm{PE}}$ of the Poisson distribution is large enough (more than $100 \mathrm{PE}$ for example), the distribution can be approximated by a 
Gaussian distribution with its mean $\mu_{\mathrm{PE}}=\lambda_{\mathrm{PE}}$ and its variance $\sigma_{\mathrm{PE}}=\sqrt{\lambda}_{\mathrm{PE}}$. Obviously,

$$
\mu_{\mathrm{PE}}=\lambda_{\mathrm{PE}}=\sigma_{\mathrm{PE}}^{2}
$$

Similar to eq. 3.1,

$$
\mu_{\mathrm{PE}}=\mu_{\mathrm{ADC}} / M_{1 \mathrm{PE}} \text { and } \sigma_{\mathrm{PE}}=\sigma_{\mathrm{ADC}} / M_{1 \mathrm{PE}},
$$

where, $\mu_{\mathrm{ADC}}$ and $\sigma_{\mathrm{ADC}}$ are the mean and variance of Gaussian distribution in units of (ADC counts $\cdot \mathrm{ns}$ ) instead of number of PE. $M_{1 \mathrm{PE}}$ can be calculated combining eq. 3.2 and eq. 3.3:

$$
\left(\mu_{\mathrm{ADC}} / M_{1 \mathrm{PE}}\right)=\left(\sigma_{\mathrm{ADC}} / M_{1 \mathrm{PE}}\right)^{2} \Longrightarrow M_{1 \mathrm{PE}}=\sigma_{\mathrm{ADC}}^{2} / \mu_{\mathrm{ADC}} .
$$

This method was used to double check $M_{1 \mathrm{PE}}$ measured from the single PE distribution shown in figure 3. The intensity of the light emitted from the LED was raised to a value that is high enough so that the minimal number of PE collected was more than 100. Several waveforms and the (ADC counts - ns) spectrum taken with this setup are shown in figure 4 . The spectrum was fitted with a Gaussian function, the mean and variance of which were used to calculate $M_{1 \mathrm{PE}}$ using eq. 3.4. $M_{1 \mathrm{PE}}$ obtained using this method is about $15 \%$ larger than that obtained from the single PE measurement. This might be partially due to the fact that the Gaussian distribution is only an approximation of the Poisson distribution, which is reflected from the large $\chi^{2}$ of the fitting as well. Nevertheless, it indicates that $M_{1 \mathrm{PE}}$ from the single PE measurement would not deviate significantly from the true value.
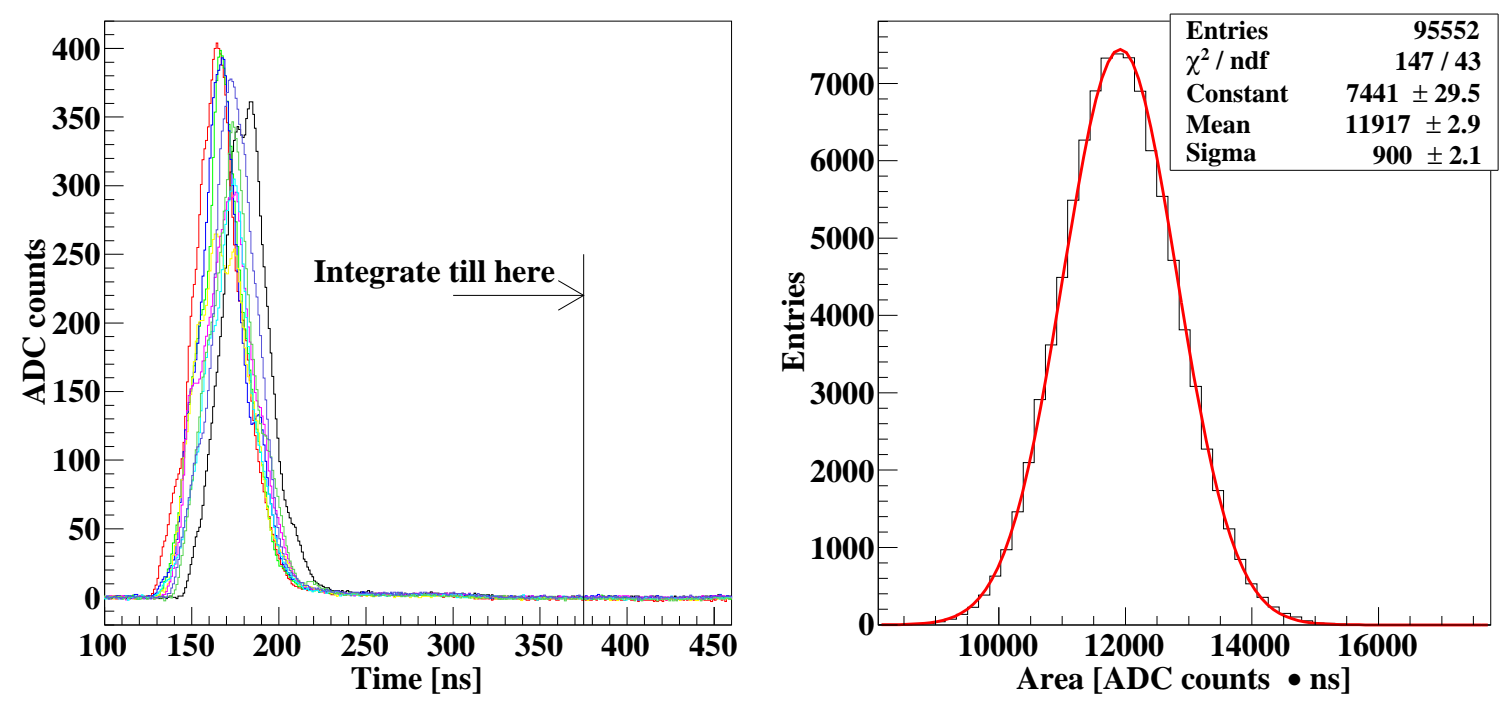

Figure 4. Left: Several consecutive waveforms taken with high LED intensity overlapped with each other. Right: Distribution of the waveforms integration fitted with a Gaussian function.

\section{Energy calibration of the system}

The energy spectra taken at room temperature with and without radioactive sources are shown in figure 5. The trigger rate for background, ${ }^{137} \mathrm{Cs},{ }^{133} \mathrm{Ba}$ and ${ }^{60} \mathrm{Co}$ data taking was $187 \mathrm{~Hz}, 280 \mathrm{~Hz}, 300 \mathrm{~Hz}$ and $330 \mathrm{~Hz}$, respectively. The $x$-axis has been converted from (ADC counts - ns) to number of PE using the method described in section 3. The spectra are normalized using the event rate above $1000 \mathrm{PE}$. The peak around $900 \mathrm{PE}$ in the 
background spectrum corresponds to the $1.46 \mathrm{MeV} \gamma$-ray radiation from ${ }^{40} \mathrm{~K}$ in the crystal. The $1.17 \mathrm{MeV}$ and $1.33 \mathrm{MeV}$ peaks of ${ }^{60} \mathrm{Co}$ can not be resolved due to the poor resolution and appears around $750 \mathrm{PE}$. The $662 \mathrm{keV}$ peak of ${ }^{137} \mathrm{Cs}$ and $356 \mathrm{keV}$ peak of ${ }^{133} \mathrm{Ba}$ can be seen around $450 \mathrm{PE}$ and $250 \mathrm{PE}$, respectively.

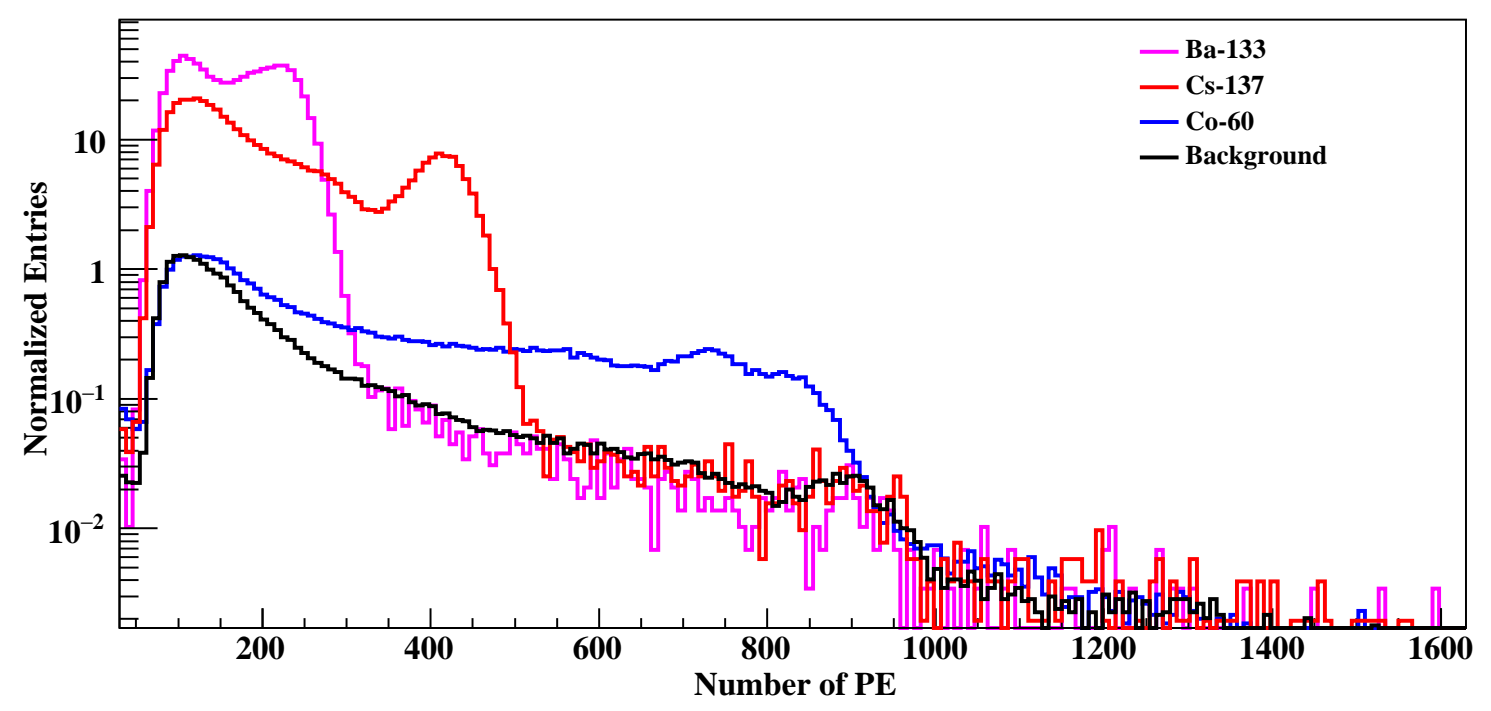

Figure 5. Energy spectra in terms of number PE at room temperature.

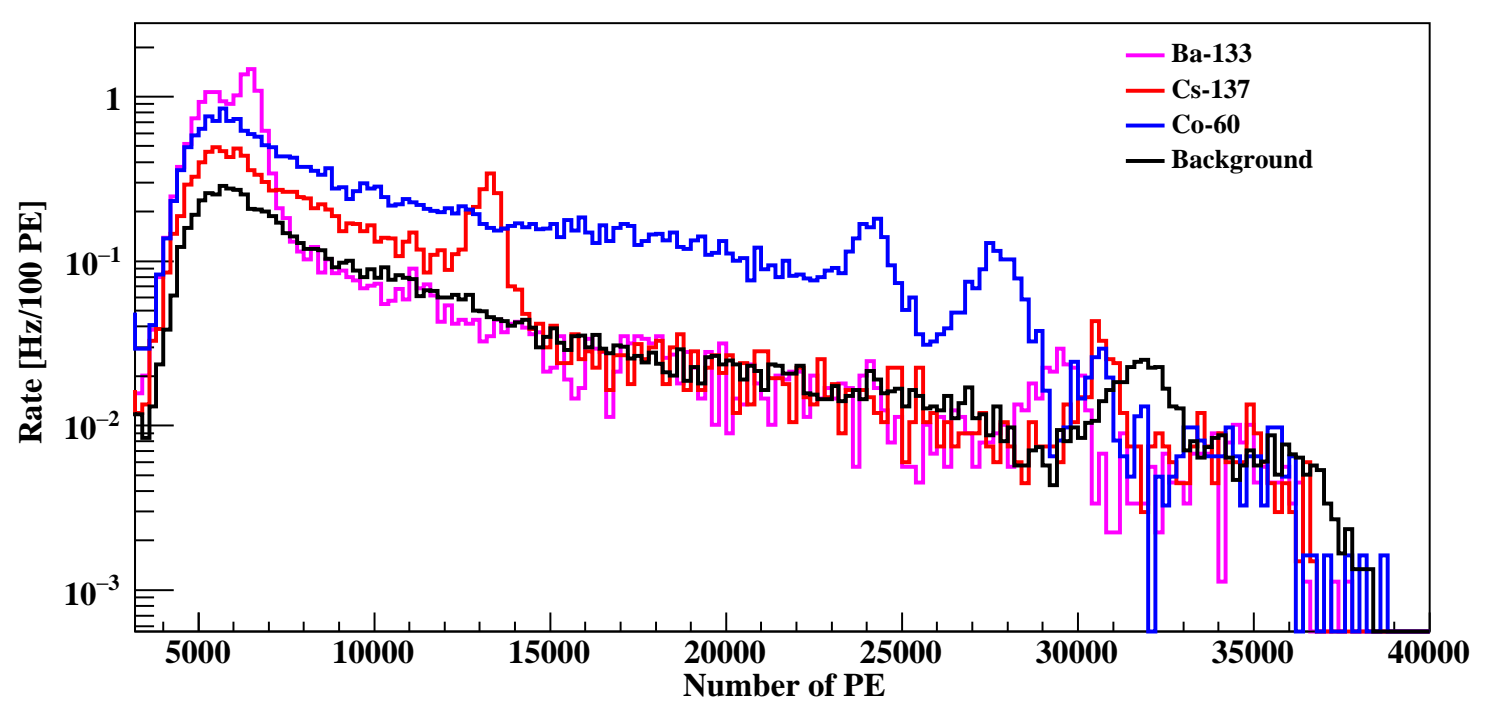

Figure 6. Energy spectra in terms of number of PE at 77 Kelvin.

The energy spectra taken at 77 Kelvin with and without radioactive sources are shown in figure 6. The trigger rate for background, ${ }^{137} \mathrm{Cs},{ }^{133} \mathrm{Ba}$ and ${ }^{60} \mathrm{Co}$ data taking was $8 \mathrm{~Hz}$, $16 \mathrm{~Hz}, 21 \mathrm{~Hz}$ and $33 \mathrm{~Hz}$, respectively. The $x$-axis has been converted from (ADC counts . ns) to number of PE using the method described in section 3. The spectra are normalized using the life times of the measurements. Compared to the spectra at room temperature, all peaks move to much higher number of $\mathrm{PE}$, indicating a dramatic improvement of the light yield. The $1.17 \mathrm{MeV}$ and $1.33 \mathrm{MeV}$ peaks of ${ }^{60} \mathrm{Co}$ are clearly separated, indicating a 
significant improvement of the energy resolution. The $1.46 \mathrm{MeV}$ peak from ${ }^{40} \mathrm{~K}$ can now be seen in all spectra. However, they do not overlap with each other perfectly, indicating an about 5\% change of the gain of the PMT during the measurement, which is reflected also in the variation of $M_{1 \mathrm{PE}}$ mentioned in section 3 .

\section{$5 \quad$ Light yield of the system}

Peaks in the energy spectra are summarized in table 2. Their positions are used to calculated the light yield of the detection system using the following equation:

$$
\text { Light yield }[\mathrm{PE} / \mathrm{keV}]=\text { Peak position [number of PE]/Peak energy }[\mathrm{keV}] \text {. }
$$

The results are shown in figure 7 . The uncertainty of each data point is mainly due to the uncertainty of $M_{1 \mathrm{PE}}$ used to convert the $x$-axes of the energy spectra from (ADC counts . ns) to number of PE.

Table 2. Summary of $\gamma$-ray peaks in the energy spectra.

\begin{tabular}{c|c|c|c|c|c}
\hline Radioactive & Energy & \multicolumn{2}{|c|}{$77 \mathrm{~K}$} & \multicolumn{2}{c}{ Room Temperature } \\
\cline { 3 - 6 } Isotopes & $(\mathrm{MeV})$ & Mean (PE) & Variance (PE) & Mean (PE) & Variance (PE) \\
\hline${ }^{133} \mathrm{Ba}$ & 0.356 & 6415.5 & 359.3 & 221.2 & 48.7 \\
${ }^{137} \mathrm{Cs}$ & 0.662 & 13222.8 & 332.3 & 413.5 & 30.7 \\
${ }^{60} \mathrm{Co}$ & 1.17 & 24110.4 & 483.8 & 729.7 & 61.8 \\
& 1.32 & 27757.3 & 543.3 & & \\
${ }^{40} \mathrm{~K}^{a}$ & 1.46 & 31750.6 & 681.2 & 895.0 & 59.0 \\
${ }^{40} \mathrm{~K}^{b}$ & & 30604.5 & 307.8 & & \\
${ }^{40} \mathrm{~K}^{c}$ & & 30690.7 & 229.0 & 887.6 & 45.2 \\
${ }^{40} \mathrm{~K}^{d}$ & & 30763.7 & 602.9 & & \\
\hline
\end{tabular}

\footnotetext{
${ }^{a}$ in background measurement

${ }^{b}$ in ${ }^{60} \mathrm{Co}$ measurement

${ }^{c}$ in ${ }^{137} \mathrm{Cs}$ measurement

${ }_{\text {in }}{ }^{133} \mathrm{Ba}$ measurement
}

The average light yield of $0.618 \pm 0.005 \mathrm{PE} / \mathrm{keV}$ and $20.4 \pm 0.8 \mathrm{PE} / \mathrm{keV}$ was obtained at room temperature and 77 Kelvin, respectively, by fitting the data points with a straight line, shown as the horizontal black line in each plot in figure 7 . The small $\chi^{2}$ of the fittings may indicate some degrees of overestimation of the uncertainty of each data point. The light yield at 77 Kelvin is about 4 times larger than that has been achieved by the KIM-CsI experiment [3], which is plotted as the red line in the right plot of figure 7 for comparison. There seems to be a systematic increase of the light yield as the energy increases as shown in the right plot of figure 7. This may indicate a slight nonlinearity in the energy response of the undoped CsI crystal at 77 Kelvin. However, limited by the large uncertainty of each data point, no quantitative conclusion can be drawn.

\section{Quantum efficiency of the PMT}

The light yield of the crystal, $\mathrm{LY}_{\text {crystal, }}$, can be calculated from the light yield of the system, $\mathrm{LY}_{\text {system, }}$, shown in the previous section, using the following equation:

$$
\mathrm{LY}_{\text {crystal }}=\mathrm{LY}_{\text {system }} / \epsilon / \mathrm{QE},
$$



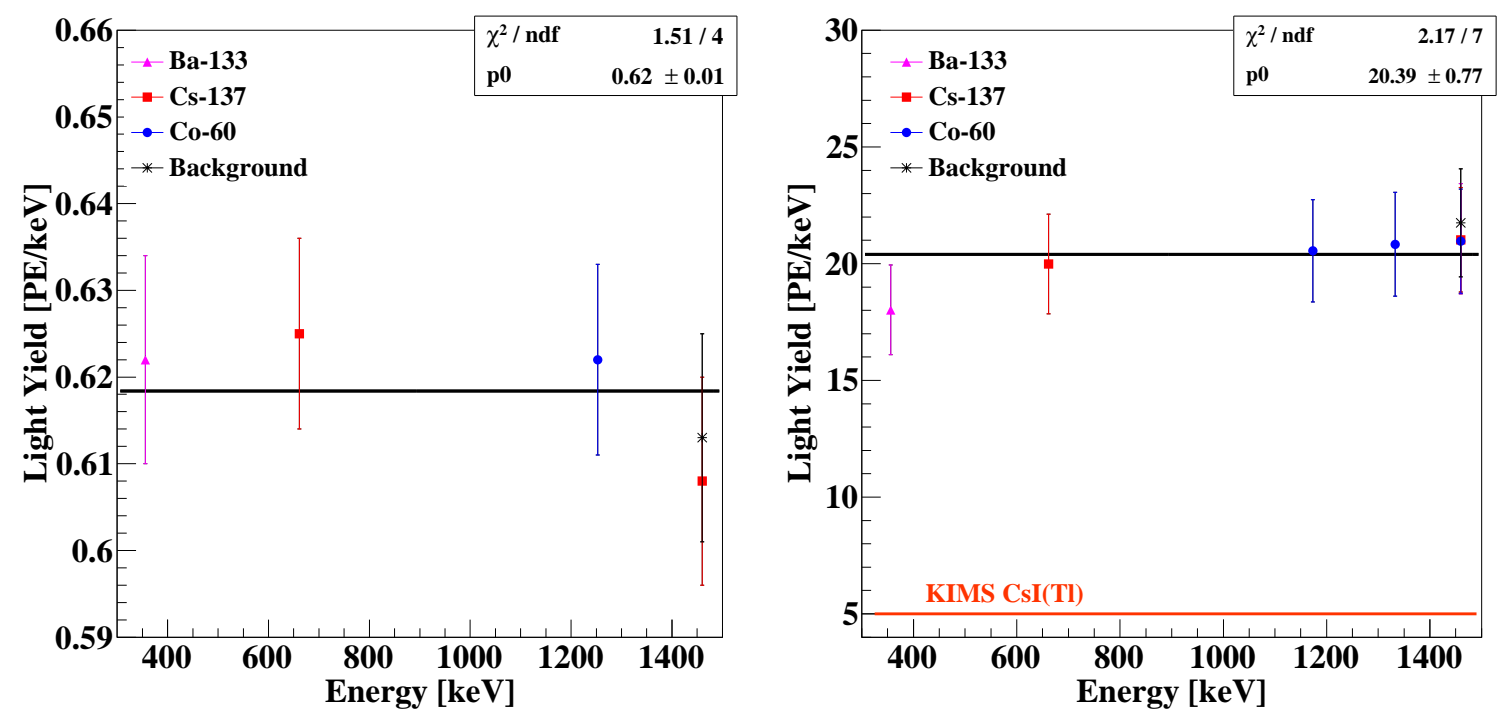

Figure 7. Light yield of the entire detection system measured at room temperature (left) and 77 Kelvin (right).

where, $\epsilon$ is the light collection efficiency, QE is the quantum efficiency of the PMT. The light collection efficiency would not change much if the reflectivity of the Teflon tape does not change much over temperature. To make sure that the increase of $L Y_{\text {system }}$ is indeed due to the increase of $L Y_{\text {crystal }}$, the stability of the QE over temperature has to be checked.

This was done by comparing the numbers of PE detected at different temperatures with a fixed LED light intensity. As shown in figure 1 , the LED ambient temperature was recorded by a PT100 sensor mounted close by. The values are summarized in table 1 . The difference is less than $10{ }^{\circ} \mathrm{C}$. The LED emission spectrum should not change much under such a small temperature variation according to the specification. The reason why the LED could be kept at room temperature while the PMT was around 77 Kelvin is because the $50 \mathrm{~cm}$ long stainless steel chamber thermally decoupled its cool bottom and warm top. This is not obvious as figure 1 is not to scale.

The result obtained with the $300 \mathrm{~nm}$ LED is shown in figure 8. The red line shows the number of $\mathrm{PE}$ distribution measured at root temperature, the black one at 77 Kelvin. The QE decreases as the tem-

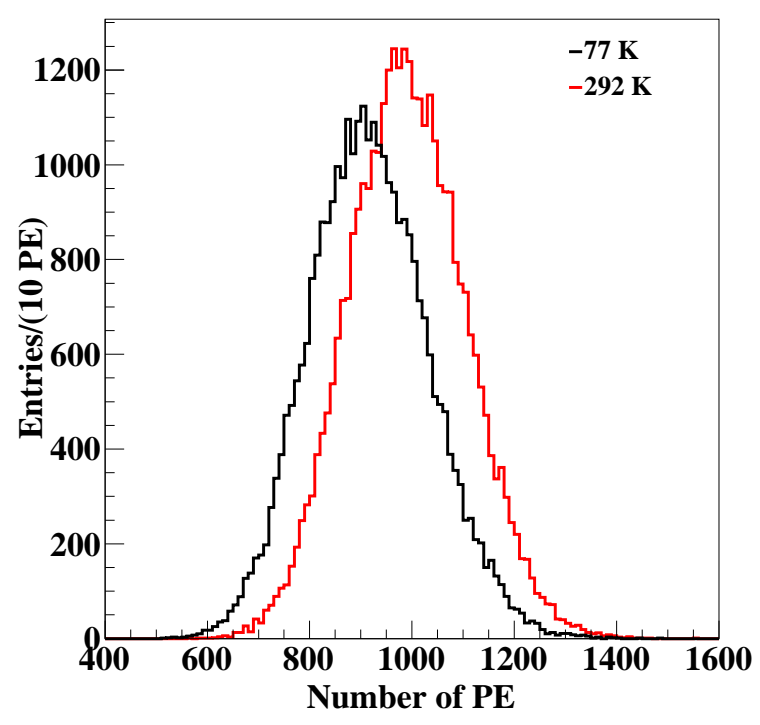

Figure 8. Number of PE detected at room temperature (red) and 77 Kelvin (black) with a fixed LED intensity. perature decreases. The relative difference between the means of the two distributions in figure 8 is $7.58 \%$. The study with the $470 \mathrm{~nm}$ LED resulted in a relative difference of $9.24 \%$. The QE of the PMT quoted by Hamamatsu Photonics K.K. at room temperature is $29.5 \%$ 
at $300 \mathrm{~nm}$. The QE at 77 Kelvin is then $29.5 \%(1-7.58 \%)=27.3 \%$. It can be seen that the increase of $L Y_{\text {system }}$ is purely due to the increase of $L Y_{\text {crystal }}$.

\section{Scintillation decay constants of the crystal}

Events in the $1.46 \mathrm{MeV}^{40} \mathrm{~K}$ peaks in the background spectra taken at both room temperature and 77 Kelvin were used to measure the scintillation decay constants of the crystal. The results were compared to those in the literature to make sure that the crystal under study was indeed an undoped CsI.

A typical light pulse taken at room temperature is shown in the left plot of figure 9 . Also shown is an exponential function,

$$
\text { Constant } \times e^{\text {Slope } \cdot x},
$$

fitted to a 15 -ns window, 3 ns after the maximum of the pulse. A sinusoidal fluctuation of $\sim 10$ ADC counts can be seen in the 45-160 ns time window and was assigned as the uncertainty to each waveform sample. The distribution of the decay constant,

$$
\tau=-1 / \text { Slope }
$$

obtained from all events is shown in the right plot of figure 9. The distribution has a mean of $15.83 \mathrm{~ns}$ and an RMS of $2.04 \mathrm{~ns}$.
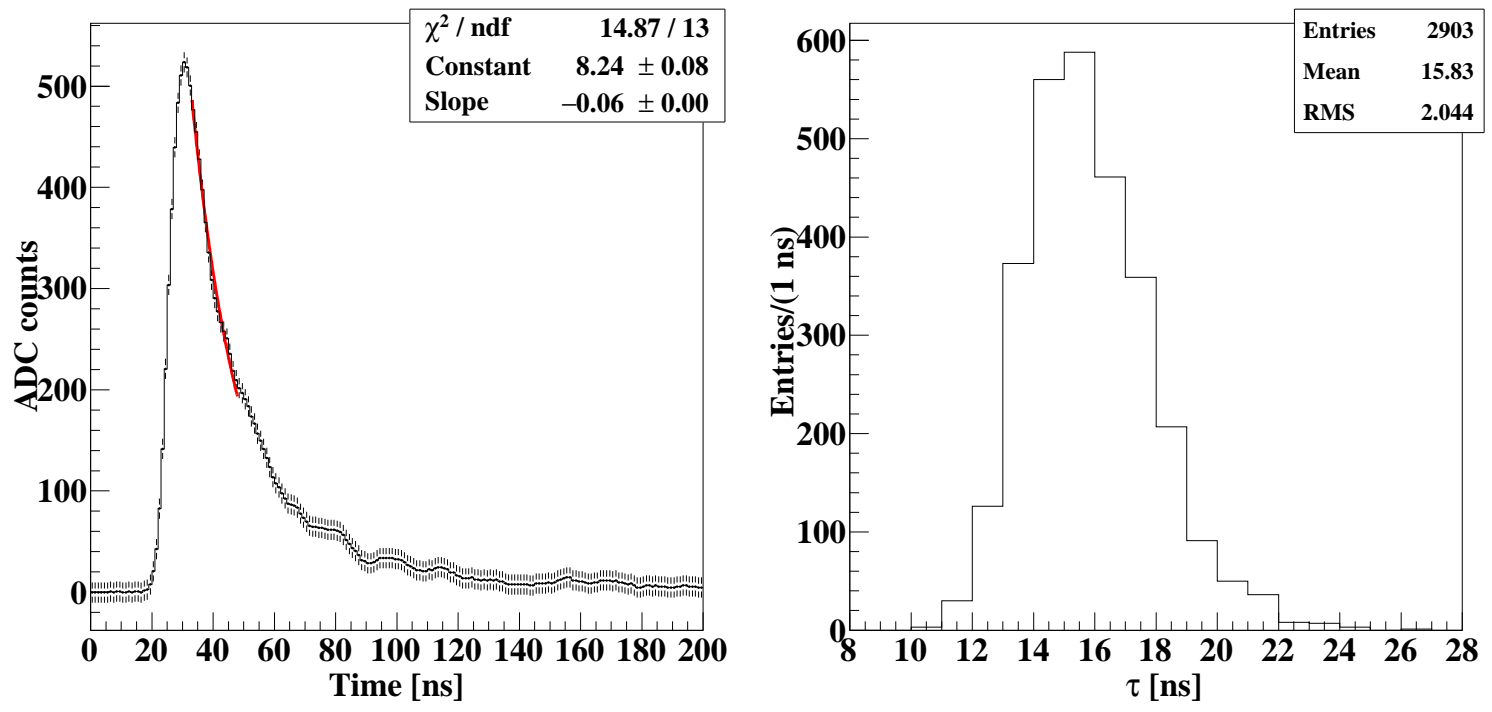

Figure 9. Left: a typical light pulse taken at room temperature fitted with eq. 7.1 to measure the decay constant. Right: distribution of the decay constant defined in eq. 7.2.

To average out the electronic noise presented in individual pulses, waveforms from 2903 events were summed up together. The averaged waveform is shown in figure 10. The standard deviation of the ADC counts of the first 25 samples is 0.26 and was used as the uncertainty of each sample in the waveform. The same exponential fitting as of figure 9 was performed and the decay constant obtained this way was $\tau=15.63 \pm 0.02 \mathrm{~ns}$, consistent with the one evaluated from individual pulses. The large $\chi^{2}$ of the fitting indicates that some systematic uncertainties have not been taken into account. Contamination from other decay components in the fitted time window may be one of them. 


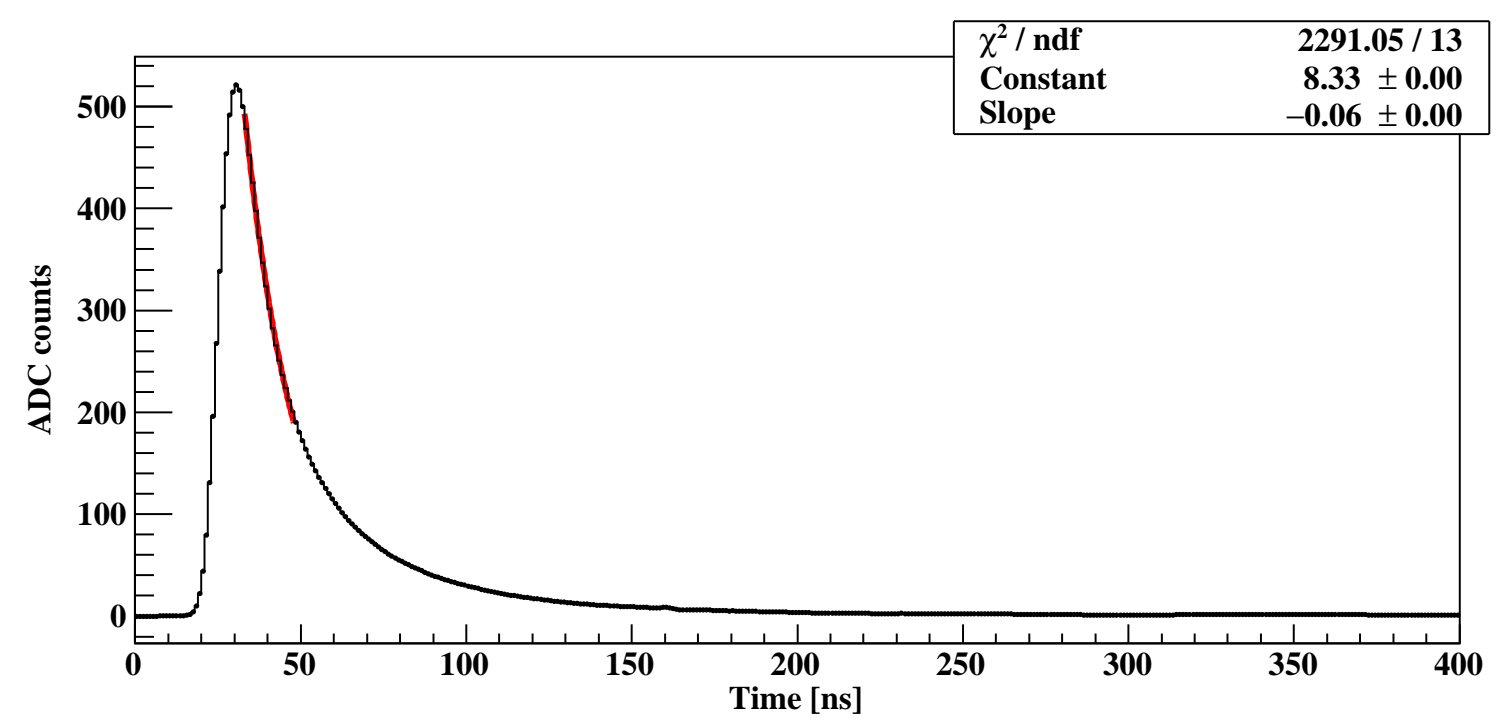

Figure 10. Averaged waveform of 2903 events at room temperature fitted with eq. 7.1.

A typical light pulse at 77 Kelvin is shown in figure 11. A fast and a slow decay component can be clearly identified and were fitted with eq. 7.1, as shown in the inlets of figure 11. The fitting window for the fast component is $7 \mathrm{~ns}, 3 \mathrm{~ns}$ after the maximum of the pulse, and the fitting window for the slow component is $700 \mathrm{~ns}, 55 \mathrm{~ns}$ after the maximum. The distributions of the two decay constants are shown in figure 12. The mean of the fast/slow component distribution is 35/1069 ns with an RMS of 11/53 ns, respectively.

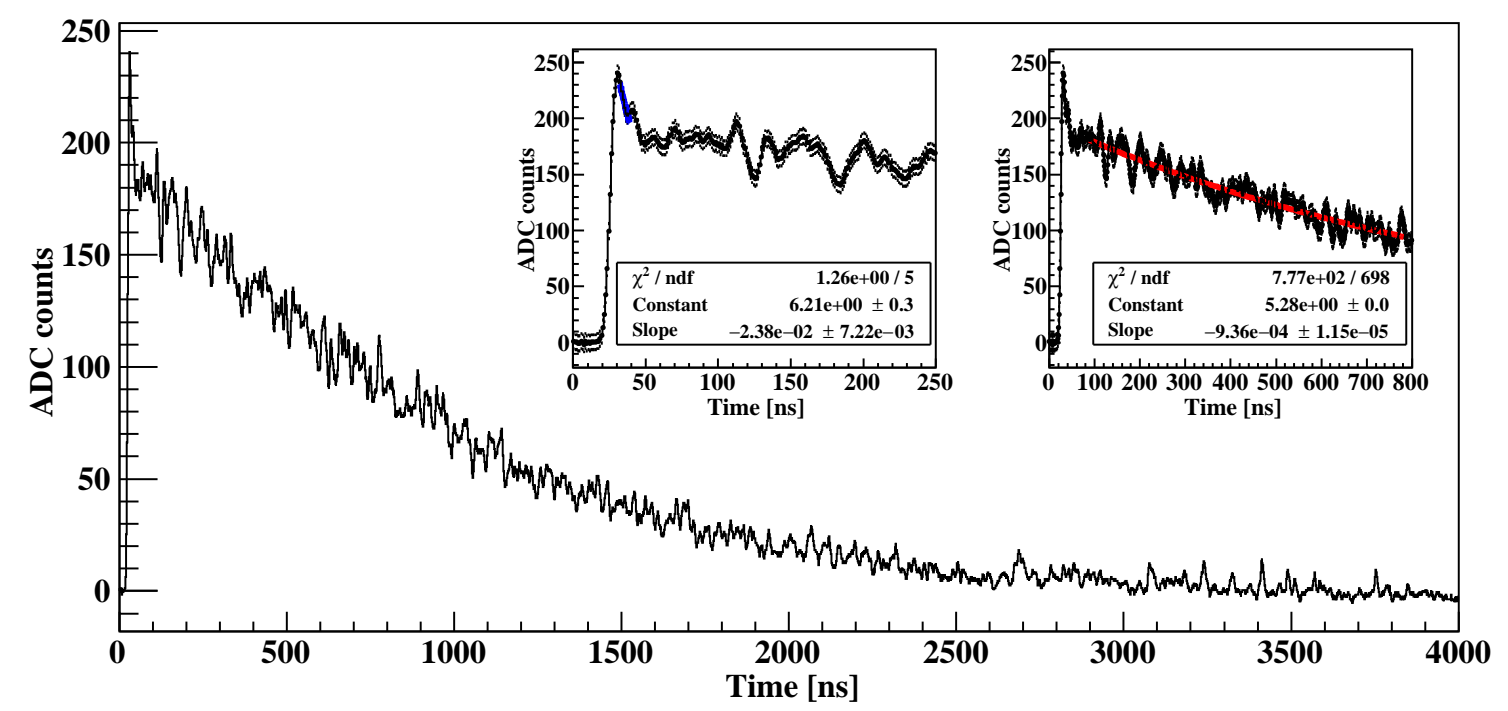

Figure 11. A typical light pulse taken at 77 Kelvin. The insets depict the fittings to the fast and slow decay components with eq. 7.1.

The average of 707 individual pulses taken at 77 Kelvin was performed similarly to that at room temperature. The averaged waveform is shown in figure 13. The standard deviation of the ADC counts of the first 25 samples is 0.6 , and was used as the uncertainty of each sample in the waveform. The same exponential fitting was performed and the fast 

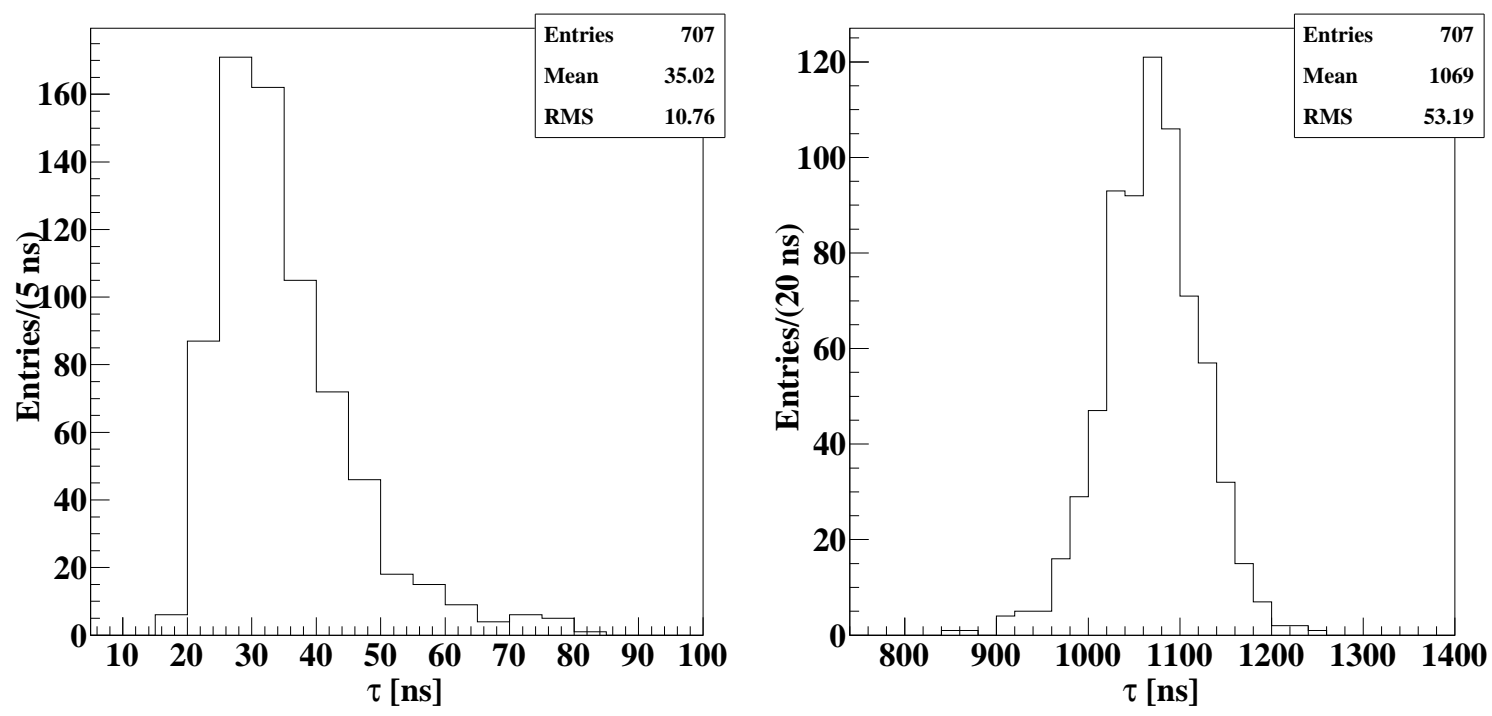

Figure 12. Distributions of the fast(left) and slow(right) decay constants at 77 Kelvin.

and slow decay constants obtained this way were $32.2 \mathrm{~ns}$ and $1092 \mathrm{~ns}$, consistent with those evaluated from individual pulses. The large $\chi^{2}$ of the fitting to the slow component indicates that some systematic uncertainties have not been taken into account. Contamination from other decay components in the fitted time window may be one of them.

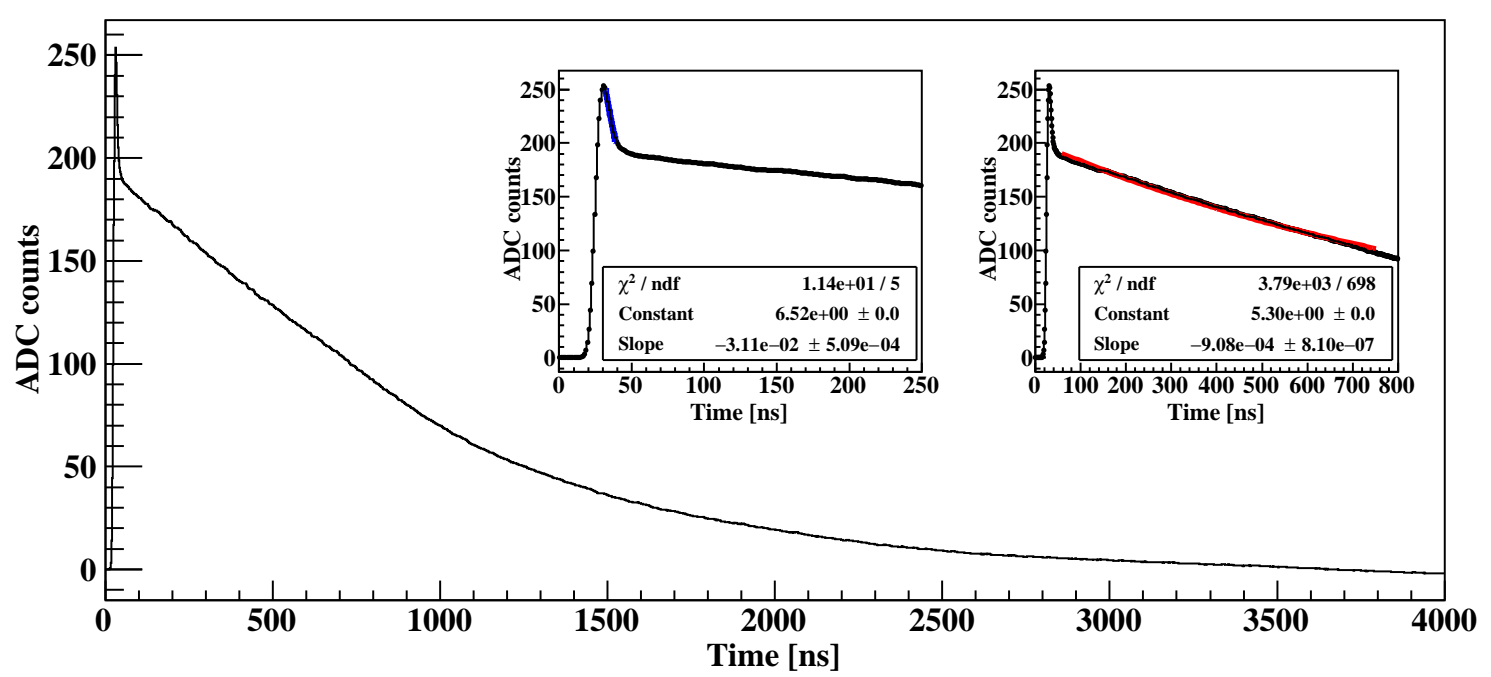

Figure 13. Averaged waveform of 800 events at 77 Kelvin. The inlets depict the exponential fittings to the fast and slow decay components with eq. 7.1.

The decay constant of the slow component, $1069 \pm 53 \mathrm{~ns}$, measured at 77 Kelvin agrees perfectly with those mentioned in ref. [21,36, 38]. It is due to the $3.7 \mathrm{eV}$ luminescence decay of self-trapped excitons in the crystal [36]. The 2 ns fast component in ref. [36] could not be observed because it was smeared out by the $15 \mathrm{~ns}$ width of single PE peaks as shown in figure 3, the $4.7 \mathrm{~ns}$ transit time spread of the PMT and the limited bandwidth of the amplifier used in the measurements. 
The decay constants at root temperature reported in various literatures are not quite consistent $[21,36,38,50,51]$. They range from 2 ns to $1 \mu \mathrm{s}$. However, all of them agree that there are several components, most of which change with temperature. The inconsistency may be explained by slight temperature difference among the experiments, and the wavelength dependence of the detection efficiency of the light sensors used in their measurements. Ref. [36] and eight references therein reported a decay constant of $15 \mathrm{~ns}$. The CsI(pure) Data Sheet provided by the company, Saint-Gobain Crystals, also reports a primary decay constant of $16 \mathrm{~ns}$ [52]. Our result is in perfect agreement with them.

\section{Impacts on rare-event search experiments}

The light yield achieved with this setup at 77 Kelvin, is about 4 times larger than that achieved in the KIMS-CsI experiment in 2014 [3], and is the largest in the world achieved with CsI to the authors' best knowledge. An energy threshold several times lower than that of KIMS-CsI can be reached assuming a similar background level. The sensitivity in the detection of dark matter particles using CsI can be significantly improved.

Since it is relatively easier to achieve a higher light collection efficiency using a smaller crystal, it makes more sense to compare the intrinsic light yield of the crystal, LY crystal, instead of the light yield of the entire detection system, $L Y_{\text {system }}$. LY $Y_{\text {crystal }}$ can be calculated using eq 6.1. Unfortunately, the light collection efficiency $\epsilon$ in the equation could not be measured with the current setup. Assuming $\epsilon=100 \%$, LY crystal can be estimated as $(20.4 \mathrm{PE} / \mathrm{keV}) / 27.3 \% \approx 75$ photons $/ \mathrm{keV}$, which is higher than that of $\mathrm{CsI}(\mathrm{Tl})$ at room temperature. Assuming $\epsilon=90 \%, \mathrm{LY}_{\text {crystal }}$ becomes

$$
(20.4 \mathrm{PE} / \mathrm{keV}) / 90 \% / 27.3 \% \approx 83 \text { photons } / \mathrm{keV} \text {. }
$$

This is about 2 times more than those of $\mathrm{NaI}(\mathrm{Tl}), \mathrm{CsI}(\mathrm{Tl})$ and $\mathrm{CsI}(\mathrm{Na})$ at room temperature, but is in agreement with the results given in ref. [21, 24, 39, 41]. Undoped CsI presents most of the advantages listed in ref. [11] for CEvNS detection. In addition, it has a higher light yield than $\mathrm{CsI}(\mathrm{Na})$ studied in ref. [11], indicating a possibly lower energy threshold. Undoped CsI at 77 Kelvin presents a fast $(2 \mathrm{~ns})$ and a slow $(1 \mu \mathrm{s})$ decay components, suggesting possible discrimination of nuclear and electronic recoil events using pulse shape analysis. No decay constant measured with neutron sources was found in the literature. However, a measurement with $\alpha$-particles did show a different decay constant of $500 \mathrm{~ns}$ [23], further suggesting the possibility.

Liquid nitrogen or argon can be used to cool an array of undoped CsI crystals directly coupled with PMTs and to shield them from external radiation at the same time. They are relatively cheap and are easy to purify. Liquid argon is also a scintillation material and can be used as an anti-coincident veto system. Such a setup is a possible alternative technique for both dark matter and CEvNS detection, providing mechanisms to lower the background level and energy threshold. Given similar behavior of undoped NaI as given in the literature $[26,27,39]$, both $\mathrm{NaI}$ and $\mathrm{CsI}$ can be deployed in such a setup, providing a comprehensive verification of the DAMA result.

It has been suggested in a recent arXiv article [53] that pure NaI and CsI at cryogenic temperature can be used to detect sub-GeV dark matter particles interacting with electrons instead of nuclei. The energy threshold can be very low if the system is allowed to be triggered by one or two scintillation photons. The major background is the dark noise of the photon sensors deployed. PMTs mentioned in our work typically have a dark count rate of several 
tens $\mathrm{Hz}$ at 77 Kelvin. If a coincident trigger of two PMTs watching the same crystal is required, the background from the dark count is negligible. This points out a much broader application of our technique than the verification of the DAMA result.

\section{Conclusion}

A light yield of $20.4 \pm 0.8 \mathrm{PE} / \mathrm{keV}$ was achieved with an undoped CsI crystal directly coupled with a PMT that works at 77 Kelvin. This is by far the largest in the world achieved with CsI crystals to the authors' best knowledge. The scintillation decay constants of the crystal were measured at both room temperature and 77 Kelvin. They agree with those in the literature, confirming the crystal used in the study was indeed an undoped CsI. Possible experimental setup has been suggested to use this technique for both dark matter and CEvNS detection.

\section{Acknowledgments}

The authors thank Christina Keller for her careful reading of manuscript. The laboratory spaces and part of equipment used for conducting this study was kindly provided by the XMASS collaboration. This work was supported by NSF PHY-1506036, the Office of Research at the University of South Dakota, the Grant-in-Aid for Encouragement of Young Scientists (B) project No. 26800122, MEXT, Japan and the World Premier International Research Center Initiative (WPI Initiative), MEXT, Japan. Computations supporting this project were performed on the High Performance Computing systems at the University of South Dakota. We thank its manager, Doug Jennewein, for providing valuable technical expertise to this project.

\section{References}

[1] DM-Ice collaboration, E. B. de Souza, J. Cherwinka, A. Cole, A. C. Ezeribe, D. Grant, F. Halzen et al., First Search for a Dark Matter Annual Modulation Signal with NaI(Tl) in the Southern Hemisphere by DM-Ice17, arXiv:1602.05939.

[2] R. Bernabei, P. Belli, F. Cappella, V. Caracciolo, S. Castellano, R. Cerulli et al., Final model independent results of DAMA/LIBRA-phase1 and perspectives of phase2, Phys. Part. Nucl. 46 (2015) 138-146.

[3] KIMS collaboration, H. S. Lee, H. Bhang, J. H. Choi, S. Choi, I. S. Hahn, E. J. Jeon et al., Search for low-mass dark matter with CsI(Tl) crystal detectors, Phys. Rev. D 90 (2014) 052006.

[4] LUX collaboration, D. S. Akerib, H. M. Araújo, X. Bai, A. J. Bailey, J. Balajthy, P. Beltrame et al., Improved Limits on Scattering of Weakly Interacting Massive Particles from Reanalysis of 2013 LUX Data, Phys. Rev. Lett. 116 (2016) 161301.

[5] SuperCDMS collaboration, R. Agnese, A. J. Anderson, T. Aramaki, M. Asai, W. Baker, D. Balakishiyeva et al., New Results from the Search for Low-Mass Weakly Interacting Massive Particles with the CDMS Low Ionization Threshold Experiment, Phys. Rev. Lett. 116 (2016) 071301.

[6] CoGent collaboration, C. E. Aalseth, P. S. Barbeau, N. S. Bowden, B. Cabrera-Palmer, J. Colaresi, J. I. Collar et al., Results from a Search for Light-Mass Dark Matter with a p-Type Point Contact Germanium Detector, Phys. Rev. Lett. 106 (2011) 131301. 
[7] J. Amaré, S. Cebrián, C. Cuesta, E. García, M. Martínez, M. A. Olivín et al., Status of the ANAIS Dark Matter Project at the Canfranc Underground Laboratory, J. Phys.: Conf. Ser. 718 (2016) 042052.

[8] H. S. Lee, The status of KIMS-NaI experiment, Nucl. Part. Phys. Proc. 273-275 (2016) 295-301.

[9] C. H. Ha, Status of the COSINE experiment, .

[10] E. Shields, J. Xu and F. Calaprice, SABRE: A New NaI(T1) Dark Matter Direct Detection Experiment, Physics Procedia 61 (2015) 169-178.

[11] J. Collar, N. Fields, M. Hai, T. Hossbach, J. Orrell, C. Overman et al., Coherent neutrino-nucleus scattering detection with a CsI[Na] scintillator at the SNS spallation source, Nucl. Instrum. Meth. A 773 (2015) 56-65.

[12] H.-T. Janka, K. Langanke, A. Marek, G. Martínez-Pinedo and B. Müller, Theory of core-collapse supernovae, Phys. Rep. 442 (2007) 38-74.

[13] J. Barranco, O. G. Miranda and T. I. Rashba, Probing new physics with coherent neutrino scattering off nuclei, J. High Energy Phys. 2005 (2005) 21.

[14] J. A. Formaggio, E. Figueroa-Feliciano and A. J. Anderson, Sterile neutrinos, coherent scattering, and oscillometry measurements with low-temperature bolometers, Phys. Rev. D 85 (2012) 013009.

[15] K. Patton, J. Engel, G. C. McLaughlin and N. Schunck, Neutrino-nucleus coherent scattering as a probe of neutron density distributions, Phys. Rev. C 86 (2012) 024612.

[16] K. M. Patton, G. C. McLaughlin and K. Scholberg, PROSPECTS FOR USING COHERENT ELASTIC NEUTRINO-NUCLEUS SCATTERING TO MEASURE THE NUCLEAR NEUTRON FORM FACTOR, Int. J. Mod. Phys. E 22 (2013) 1330013.

[17] A. Drukier and L. Stodolsky, Principles and applications of a neutral-current detector for neutrino physics and astronomy, Phys. Rev. D 30 (1984) 2295-2309.

[18] TEXONO collaboration, S. Kerman, V. Sharma, M. Deniz, H. Wong, J. W. Chen, H. Li et al., Coherency in Neutrino-Nucleus Elastic Scattering, Phys. Rev. D 93 (2016), [arXiv: 1603.08786].

[19] D. Y. Akimov, A. K. Berdnikova, V. A. Belov, A. I. Bolozdynya, A. A. Burenkov, Y. V. Efremenko et al., RED-100 detector for the first observation of the elastic coherent neutrino scattering off xenon nuclei, J. Phys.: Conf. Ser. 675 (2016) 012016.

[20] COHEREnT collaboration, D. Akimov, P. An, C. Awe, P. S. Barbeau, P. Barton, B. Becker et al., The COHERENT Experiment at the Spallation Neutron Source, arXiv:1509.08702.

[21] J. Bonanomi and J. Rossel, Scintillations de luminescence dans les iodures d'alcalins, Helv. Phys. Acta 25 (1952) 725.

[22] E. Hahn and J. Rossel, Scintillations des particules dans Csl, Helv. Phys. Acta 26 (1953) 271.

[23] B. Hahn and J. Rossel, Scintillations dans Csl et spectrométrie, Helv. Phys. Acta 26 (1953) 803.

[24] W. Van Sciver, Alkali Halide Scintillators, IRE Trans. Nucl. Sci. 3 (1956) 39-50.

[25] L. E. Beghian, G. H. R. Kegel and R. P. Scharenberg, Fast Sodium Iodide Spectrometer and Its Application to Millimicrosecond Time Measurement, Rev. Sci. Instrum. 29 (1958) 753.

[26] W. J. Van Sciver and L. Bogart, Fundamental Studies of Scintillation Phenomena in NaI, IRE Trans. Nucl. Sci. 5 (1958) 90-92.

[27] W. Van Sciver, Fluorescence and Reflection Spectra of NaI Single Crystals, Phys. Rev. 120 (1960) 1193-1205. 
[28] J. Hsu and W. C. Kaiser, Quantum Statistical Treatment of Relative Light Intensity of NaI (Pure) Crystal as a Function of Absolute Temperature, IEEE Trans. Nucl. Sci. 13 (1966) 704-715.

[29] M. P. Fontana, H. Blume and W. J. van Sciver, Properties of Exciton States in NaI I. The Intrinsic Photoluminescence, Physica Status Solidi (b) 29 (1968) 159-166.

[30] J. B. West and A. J. L. Collinson, The low temperature scintillation response of unactivated sodium iodide to gamma-rays, J. Phys. B 3 (1970) 1363-1373.

[31] M. P. Fontana and W. J. van Sciver, Quenched-in Photoluminescent Color Centers in NaI, Physica Status Solidi (b) 37 (1970) 375-380.

[32] W. L. Emkey, P. V. Meyers and W. J. Van Sciver, Anisotropic intrinsic photoluminescence in NaI, Journal of the Optical Society of America 66 (1976) 264.

[33] D. E. Persyk, M. A. Schardt, T. E. Moi, K. A. Ritter and G. Muehllehner, Research on Pure Sodium Iodide as a Practical Scintillator, IEEE Trans. Nucl. Sci. 27 (1980) 167-171.

[34] C. Woody, P. Levy, J. Kierstead, T. Skwarnicki, Z. Sobolewski, M. Goldberg et al., Readout techniques and radiation damage of undoped cesium iodide, IEEE Trans. Nucl. Sci. 37 (1990) 492-499.

[35] R. Williams and K. Song, The self-trapped exciton, J. Phys. Chem. Solids 51 (1990) 679-716.

[36] H. Nishimura, M. Sakata, T. Tsujimoto and M. Nakayama, Origin of the 4.1-eV luminescence in pure CsI scintillator, Phys. Rev. B 51 (1995) 2167-2172.

[37] J. Wear, J. Karp, A. Haigh and R. Freifelder, Evaluation of moderately cooled pure NaI as a scintillator for position-sensitive PET detectors, IEEE Trans. Nucl. Sci. 43 (1996) 1945-1951.

[38] C. Amsler, D. Grögler, W. Joffrain, D. Lindelöf, M. Marchesotti, P. Niederberger et al., Temperature dependence of pure CsI: scintillation light yield and decay time, Nucl. Instrum. Meth. A 480 (2002) 494-500.

[39] M. Moszyński, M. Balcerzyk, W. Czarnacki, M. Kapusta, W. Klamra, P. Schotanus et al., Study of pure NaI at room and liquid nitrogen temperatures, IEEE Trans. Nucl. Sci. 50 (2003) 767-773.

[40] M. Moszyński, W. Czarnacki, W. Klamra, M. Szawlowski, P. Schotanus and M. Kapusta, Application of large area avalanche photodiodes to study scintillators at liquid nitrogen temperatures, Nucl. Instrum. Meth. A 504 (2003) 307-312.

[41] M. Moszyński, M. Balcerzyk, W. Czarnacki, M. Kapusta, W. Klamra, P. Schotanus et al., Energy resolution and non-proportionality of the light yield of pure CsI at liquid nitrogen temperatures, Nucl. Instrum. Meth. A 537 (2005) 357-362.

[42] M. Moszyński, W. Czarnacki, A. Syntfeld-Kazuch, A. Nassalski, T. Szczȩśniak, L. Swiderski et al., A Comparative Study of Undoped NaI Scintillators With Different Purity, IEEE Trans. Nucl. Sci. 56 (2009) 1655-1660.

[43] P. Sibczyński, M. Moszyński, T. Szczȩśniak, W. Czarnacki, A. Syntfeld-Kazuch and P. Schotanus, Further study of undoped NaI scintillators with different purity, in IEEE Nucl. Sci. Sym. Med. Imag. Conf., pp. 574-579, IEEE, 2010.

[44] P. Sibczyński, M. Moszyński, T. Szczȩśniak and W. Czarnacki, Study of NaI(Tl) scintillator cooled down to liquid nitrogen temperature, JINST 7 (2012) P11006-P11006.

[45] C. Sailer, B. Lubsandorzhiev, C. Strandhagen and J. Jochum, Low temperature light yield measurements in NaI and NaI(Tl), Europ. Phys. J. C 72 (2012) 2061.

[46] G. Collazuol, "The SiPM Physics and Technology - a Review." https://indico.cern.ch/ event/164917/contributions/1417121/attachments/198512/278663/PhotoDet12_- _ collazuol_-_v3.pdf. 
[47] M. Yamashita. http://w4.gakkai-web.net/jps_search/2010au/u.html\#13aSG-1-1.

[48] A. Minamino, K. Abe, Y. Ashie, J. Hosaka, K. Ishihara, K. Kobayashi et al., Self-shielding effect of a single phase liquid xenon detector for direct dark matter search, Astropart. Phys. 35 (2012) 609-614.

[49] Y. Hotta, "Latest developments in PMTs for low temperature operation." http://www.pa. ucla.edu/sites/default/files/webform/Hamamatsu\%20Presentation\%20f or\%20DM2014. pdf.

[50] S. Kubota, S. Sakuragi, S. Hashimoto and J.-z. Ruan, A new scintillation material: Pure CsI with 10 ns decay time, Nucl. Instrum. Meth. A 268 (1988).

[51] P. Schotanus and R. Kamermans, Scintillation characteristics of pure and Tl-doped CsI crystals, IEEE Trans. Nucl. Sci. 37 (1990) 177-182.

[52] "CsI Pure Data Sheet." http://www.crystals.saint-gobain.com/uploadedFiles/SGCrystals/Documents/CsI\%20Pure\%20Data\%20Sheet.pdf.

[53] S. Derenzo, R. Essig, A. Massari, A. Soto and T.-T. Yu, Direct Detection of sub-GeV Dark Matter with Scintillating Targets, arXiv:1607.01009. 